\title{
Correction to: Effect of the internal degrees of freedom of the gas molecules on the heat and mass transfer in long circular capillaries
}

\author{
Christos Tantos $^{1} \cdot$ Stylianos Varoutis ${ }^{1} \cdot$ Christian Day $^{1}$
}

Published online: 2 April 2021

○) Springer-Verlag GmbH Germany, part of Springer Nature 2021

\section{Correction to: Microfluid Nanofluid (2020) 24:95 https://doi.org/10.1007/s10404-020-02400-z}

The original publication of the article contains typographical errors that occurred during the typesetting stage of the article, which need to be amended as mentioned below and they do not affect any of the reported numerical results.

1. In Eq. (2), the vectors $\mathbf{F}, \mathbf{F}^{(t)}, \mathbf{F}^{(t, r)}$ and $\mathbf{F}^{(t, r, v)}$ should be written in bold-italics, i.e. $\boldsymbol{F}, \boldsymbol{F}^{(t)}, \boldsymbol{F}^{(t, r)}$ and $\boldsymbol{F}^{(t, r, v)}$.

2. In Eqs. (9), (14), (15), and (17), the standard function "sin" should not be written in italic font, i.e. $\sin \theta$.

3. The text immediately after Eq. (30) should read as: "where $\mu$ is the dynamic viscosity,..."

4. After Eq. (30), in the last sentence of the paragraph text should read as: "The parameters $\rho D_{\text {rot }} / \mu, A^{*}$ and $Z_{\text {rot }}$ can be found in the work performed by (Mason and Uribe 1996) as functions of temperature for various polyatomic gases."

5. In Sect. 3.1, in line 9 of the fourth paragraph the inline equation " $\lambda_{\text {rot }}=0.892$ " should be replaced by " $Z_{\text {rot }}=1$ ".

6. In Sect. 3.1, at the end of the fifth paragraph the paragraph text should be read as: "...the relaxation parameter $\lambda . "$

7. In Sect. 3.1, in line 8 of the sixth paragraph the paragraph text should be read as: "...the parameters $\omega_{0}, \omega_{1}$ and $\kappa$ with those used in the calculations of $G^{(T)}$."

The original article can be found online at https://doi.org/10.1007/ s10404-020-02400-z.

Christos Tantos

christos.tantos@kit.edu

Institute for Technical Physics, Karlsruhe Institute of Technology, Hermann-von-Helmholtz-Platz 1, 76344 Eggenstein-Leopoldshafen, Germany
8. In Sect. 3.2, in line 16 of the first paragraph the inline equation " $\lambda_{\text {rot }}=0.656$ " should be replaced by " $\lambda=2 / 3$ ".

9. In Sect. 3.2, in the line 15 of the third paragraph the inline parameter " $\lambda_{\mathrm{tr}}$ " should be replaced by " $\lambda_{\mathrm{tr}}$ ".

10. In Sect. 3.2, in the line 25 of the third paragraph the inline expression " $100 \times\left(3 \lambda_{t r}-2\right) / 3 \lambda_{t r}$ " should be replaced by " $100 \times\left(3 \lambda_{t r}-2\right) / 3 \lambda_{t r}$ ".

11. In Sect. 3.2, in the line 2 of the fourth paragraph the phrase "..the computational resources are limited to the results...", should be replaced by "..the computational resources are limited, the results...".

12. In Sect. 3.2, before Eq. (34), in line 12 of the sixth paragraph the phrase "It should be mentioned that as in the case of $Q_{\text {rot }}^{(T)}, \ldots$, should be replaced by "It should be mentioned that symmetrically to the case of $Q_{\mathrm{rot}}^{(T)}$, ...".

Publisher's Note Springer Nature remains neutral with regard to jurisdictional claims in published maps and institutional affiliations. 